\title{
Broadband phase-coherent optical frequency synthesis with actively linked Ti:sapphire and Cr:forsterite femtosecond lasers
}

\author{
Albrecht Bartels, Nathan R. Newbury, Isabell Thomann, Leo Hollberg, and Scott A. Diddams \\ National Institute of Standards and Technology, 325 Broadway, Boulder, Colorado 80305
}

\begin{abstract}
We link the output spectra of a Ti:sapphire and a Cr:forsterite femtosecond laser phase coherently to form a continuous frequency comb with a wavelength coverage of $0.57-1.45 \mu \mathrm{m}$ at power levels of $1 \mathrm{nW}$ to $40 \mu \mathrm{W}$ per frequency mode. To achieve this, the laser repetition rates and the carrier-envelope offset frequencies are phase locked to each other. The coherence time between the individual components of the two combs is $40 \mu \mathrm{s}$. The timing jitter between the lasers is $20 \mathrm{fs}$. The combined frequency comb is self-referenced for access to its overall offset frequency. We report the first demonstration to our knowledge of an extremely broadband and continuous, high-powered and phase-coherent frequency comb from two femtosecond lasers with different gain media.
\end{abstract}

Extremely broadband femtosecond pulse sources with well-defined carrier-envelope phase evolution have been the subject of intense research over the past few years. ${ }^{1-3}$ The time-domain motivation for the push toward increased bandwidth is the generation of optical pulses that approach the single-cycle regime and can be used for phase-sensitive spectroscopy and nonlinear experiments. ${ }^{4}$ The frequency-domain motivation is an interest in extending the bandwidth over which optical frequencies can be precisely synthesized and subsequently used for absolute optical frequency measurements or frequency comparison. ${ }^{1,5}$ As was demonstrated by Shelton et al. ${ }^{6}$ with two Ti:sapphire lasers operating near $800 \mathrm{~nm}$, an attractive way to overcome the bandwidth limitations of current systems is by phase-coherent linking of two mode-locked laser sources. A necessary ingredient of such work is tight phase locking of the repetition rates (i.e., the frequency comb spacing) of two lasers. This has now been demonstrated with picosecond ${ }^{7-8}$ and femtosecond ${ }^{10,11}$ lasers based on different gain media. In these sources, however, the absolute positions (or offsets) of the two frequency combs still fluctuate independently. Using nonlinear frequency conversion to compare widely separated frequency combs, others have shown that a stable fractional relationship between the absolute positions of the two frequency combs can be established. ${ }^{12-14}$ However, if in such a case the frequency offset of at least one of the combs is not known and fixed $a$ priori, then the difference in the offsets of the two combs is still not fixed. Here we take a further step and heterodyne the overlapping spectral extremes of the combs emitted by broadband Ti:sapphire and Cr:forsterite femtosecond lasers, thereby directly gaining access to the difference in the offset frequencies, $\Delta f_{0}=f_{0, \mathrm{Ti}} \quad f_{0, \mathrm{Cr}}{ }^{11}$ This difference is then phase locked to zero to create a single phase-coherent frequency comb (extending from 0.57 to $1.45 \mu \mathrm{m}$ ) with uniform spacing. We measure its overall offset frequency (which we call $F_{0}$ ) by beating the second harmonic of light near $1.28 \mu \mathrm{m}$ against a visible portion of the fundamental frequency comb near $0.64 \mu \mathrm{m}$.
The broadband Ti:sapphire laser and the Cr:forsterite laser that we employ were described elsewhere. ${ }^{15,16}$ Their output spectra are shown in Fig. 1. The combined coverage extends from 0.57 to $1.45 \mu \mathrm{m}$ at a power level of $1 \mathrm{nW}$ per frequency mode, a level that is generally sufficient for frequency metrology applications. It is important to point out that the spectra overlap without external spectral broadening. The ring oscillators have a repetition rate of $433 \mathrm{MHz}$. Their output powers are both approximately $500 \mathrm{~mW}$. Both lasers have one mirror mounted onto a piezoelectric transducer (PZT) by which we control their repetition rates. Acousto-optic modulators (AOMs) in the pump laser beams allow us to control the offset frequencies independently.

To create a combined phase-coherent frequency comb from the two lasers we employ the scheme sketched in Fig. 2. We equalize the mode spacings of the combs by phase locking the repetition rate of the Ti:sapphire laser, $f_{R}$, Ti, to that of the Cr:forsterite laser, $f_{R, \mathrm{Cr}}$. The phase lock is based on a nonlinear cross-correlation signal between the laser outputs that is generated in a type I phase-matched $\beta$-barium borate $(\mathrm{BBO})$ crystal in a noncollinear configuration. With appropriate relative delay between the two pulse trains of one-half pulse width, the slope of this cross-correlation signal is approximately linear and serves as the error signal in our feedback loop. It is

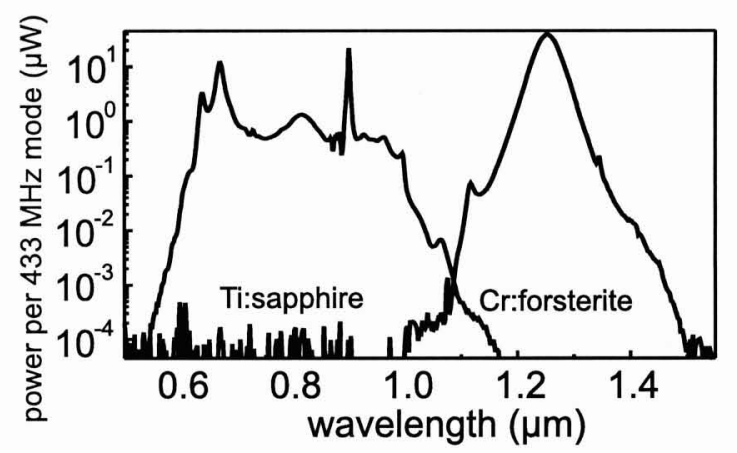

Fig. 1. Spectra of the Ti:sapphire and Cr:forsterite lasers. 


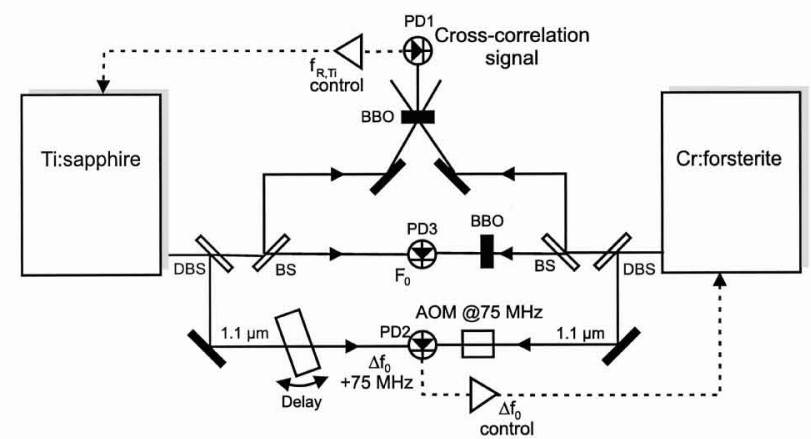

Fig. 2. Setup for phase locking the Ti:sapphire and Cr:forsterite lasers. A cross-correlation signal is generated in a BBO crystal that is detected with photodiode PD1. This signal is fed back to the PZT in the Ti:sapphire laser. The portions of the spectra that are reflected by a DBS near $1.1 \mu \mathrm{m}$ are combined in photodiode PD2 to generate a heterodyne beat signal at $\Delta f_{0}$. A thick quartz plate adjusts the relative delay between the pulses. Light from the Cr:forsterite laser is frequency doubled and generates a heterodyne beat $F_{0}$ with the output of the Ti:sapphire laser on photodiode PD3. DBSs, dichroic beam splitters; BSs, beam splitters.

offset from zero, filtered, and amplified, and it drives the PZT that holds a cavity mirror of the Ti:sapphire laser. The advantage of this method compared to conventional methods that employ photodetection of the pulse trains and phase-locking electronic microwave harmonics of the repetition rate is that the error signal has an inherently higher phase sensitivity at the lock point (approximately $100 \mathrm{fs} / \mathrm{V}$, compared with $100 \mathrm{ps} / \mathrm{V}$ ) and therefore allows for tighter phase locking. Figure 3(a) shows a spectrum of the timing fluctuations between the two locked lasers as measured with an out-of-loop nonlinear crosscorrelator (not shown in Fig. 2). It exhibits a pronounced peak above $10 \mathrm{kHz}$ that cannot be canceled by our feedback loop because of the limited bandwidth of the PZT. Compared to what was reported in Ref. 17 for phase-locked Ti:sapphire femtosecond lasers, this pronounced timing noise at frequencies of tens of kilohertz is quite unusual. We believe that it is due to excess power noise in the same frequency regime that is present in the Yb:glass fiber laser that pumps the Cr:forsterite laser. This noise is transferred onto the Cr:forsterite laser and is likely converted into timing noise of its output pulses [Fig. 3(b)]. The disagreement between the peak positions of the timing jitter and amplitude noise spectra is due to a resonance in our timing jitter feedback loop at $\approx 30 \mathrm{kHz}$. The integrated timing jitter between the two lasers is $20 \mathrm{fs}$ in a bandwidth from $1 \mathrm{~Hz}$ to $100 \mathrm{kHz}$, which is sufficient to retain a large enough pulse overlap that measurement of $\Delta f_{0}$ and $F_{0}$ is feasible. However, under the assumption that amplitude-to-timing noise conversion in the Cr:forsterite laser is our dominant source of high-frequency timing jitter, Fig. 3 suggests that elimination of high-frequency amplitude noise in the Yb:glass fiber laser could yield a timing jitter of $2 \mathrm{fs}$ in a $100-\mathrm{kHz}$ bandwidth. This reduction in timing jitter would be desirable because it would also immediately reduce the phase noise of the heterodyne beat signals discussed in the following paragraphs.

With the repetition rates of the two lasers locked, we measured the difference of the lasers' carrierenvelope-offset frequencies $\Delta f_{0}$. We picked the overlapping portions of the two laser spectra at $1.1 \mu \mathrm{m}$ with dichroic beam splitters. Subsequently the $\mathrm{Cr}$ :forsterite frequency comb near $1.1 \mu \mathrm{m}$ was offset by $75 \mathrm{MHz}$ with an $\mathrm{AOM}$ driven at $f_{\mathrm{AOM}}=75 \mathrm{MHz}$. A heterodyne beat with frequency $f_{b}=\Delta f_{0}+f_{\text {AOM }}$ was detected with a low-noise InGaAs photodiode, provided that the pulses were temporally overlapped by rotation of a thick quartz plate in the Ti:sapphire beam path. Approximately $10 \mu \mathrm{W}$ of power from each laser contributed to the beat signal, which had a $35-\mathrm{dB}$ signal-to-noise ratio at a $300-\mathrm{kHz}$ resolution bandwidth.

To make $f_{0, \mathrm{Cr}}=f_{0 \text {, Ti }}$ (i.e., $\Delta f_{0}=0 \mathrm{~Hz}$ ) we phase lock $f_{b}$ to $f_{\mathrm{AOM}}$ by using a digital phase comparator to generate an error signal that controls the pump power of the Cr:forsterite laser with the AOM. This approach makes it possible to lock $\Delta f_{0}$ to zero while circumventing problems related to the low-frequency noise floor of the photodetector in this feedback loop. To test the stability of this phase lock we counted $f_{b}$ at a 1-s gate time and derived from this a time record of the deviations of $\Delta f_{0}$ from $0 \mathrm{~Hz}$. Figure 4 (a) shows that the deviations are on a $10-\mathrm{mHz}$ scale, limited by the resolution of the frequency counter. This proves that the combined frequency comb is readily suitable for precise optical frequency measurements. To characterize the tightness of this phase lock, we measured the phase
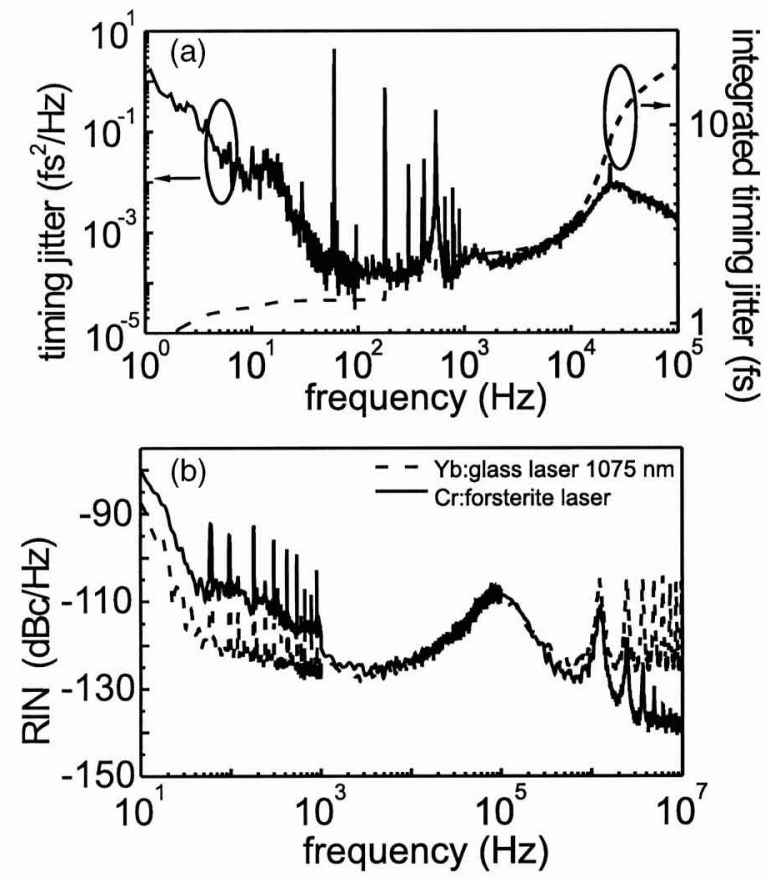

Fig. 3. (a) Out-of-loop spectral density of the timing jitter between the pulse trains from the Ti:sapphire and the Cr:forsterite lasers with the feedback loop engaged (solid curve, left-hand scale). Integrated timing jitter versus integration bandwidth (dashes curve, right-hand scale). (b) Relative intensity noise (RIN) spectral density of the Cr:forsterite laser and its pump laser. 

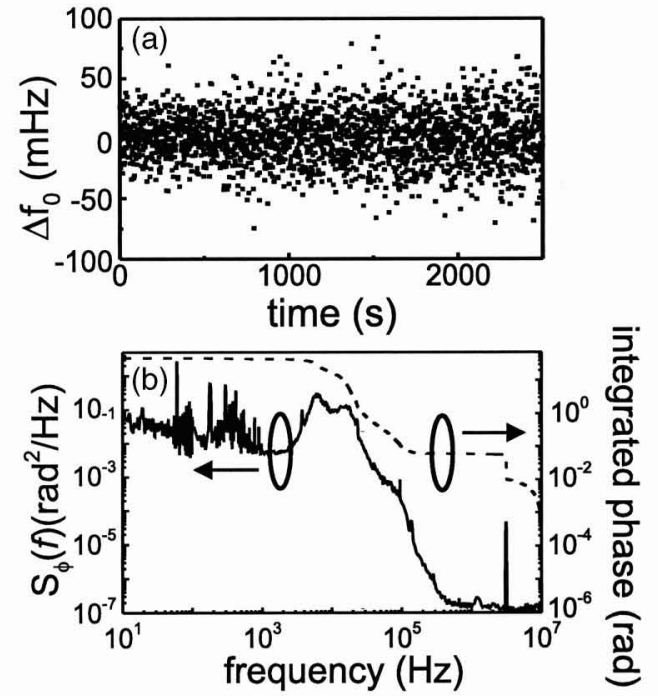

Fig. 4. (a) Record of consecutive frequency measurements of the phase-locked $\Delta f_{0}$ at a 1-s gate time. The standard deviation is $20 \mathrm{mHz}$. (b) Phase-noise spectrum of the signal at $f_{b}$ (also that of $\Delta f_{0}$ ), equal to the relative phase noise of the equally spaced optical frequency combs (solid curve). The dashed curve is the integral of $S_{\phi}(f)$, as mentioned in the text.

noise spectrum $S_{\phi}(f)$ of $f_{b}$, which is equal to the phase noise of $\Delta f_{0}$ and the relative phase noise of the equally spaced optical frequency combs. The result is shown in Fig. 4(b). The spectrum again shows strong excess noise at a few tens of kilohertz that is likely due to the timing jitter among the lasers that broadens $f_{b}$ by introducing both amplitude and phase noise. Integration of the phase noise spectrum $\int_{10 \mathrm{MHz}}^{f_{l}} S_{\phi}(f) \mathrm{d} f$ shows that $1 \mathrm{rad}$ is reached at $f_{l}=27 \mathrm{kHz}$, from which we infer a coherence time of the lock between our lasers of $\approx 40 \mu \mathrm{s}$. In other words, at this stage of the experiment, bunches of $\approx 15,000$ successive pulses from the two lasers are truly phase coherent. The phase coherence that we achieved appears to be similar to that which was reported by Shelton et al. when integration times of as low as $20 \mu$ s were used to produce high-contrast interference between two phase-locked Ti:sapphire lasers. ${ }^{6}$

To facilitate an absolute frequency measurement or ultimately to control the carrier-envelope phase of a combined field, however, we need to measure $F_{0}=$ $2 f_{0, \mathrm{Cr}} \quad f_{0, \mathrm{Ti}}=f_{0, \mathrm{Cr}}=f_{0, \mathrm{Ti}}$. To do so, we employ the conventional $f \quad 2 f$ self-referencing method. ${ }^{1}$ A portion of the Cr:forsterite laser is frequency doubled in a type I phase-matched BBO crystal and beats against the spectrally matching portion of the fundamental Ti:sapphire spectrum to generate a signal at $F_{0}$. The power that contributes to this beat is $\approx 1 \mathrm{~mW}$ for each laser, and a signal-to-noise ratio that exceeds $40 \mathrm{~dB}$ in a $300-\mathrm{kHz}$ resolution bandwidth is achieved. Thus far, we have succeeded in frequency locking $F_{0}$. However, our attempts to phase lock $F_{0}$ have not yet been successful.

In conclusion, we have tightly phase locked the repetition rates of a Ti:sapphire and a Cr:forsterite femtosecond laser at $433 \mathrm{MHz}$. Subsequently we measured the offset $\Delta f_{0}$ between the equally spaced frequency combs and were able to stabilize it to $0 \mathrm{~Hz}$. For the first time to our knowledge, we have thereby created a continuous, high-powered, and phasecoherent frequency comb from two femtosecond lasers with different gain media. A heterodyne beat between the second harmonic of the Cr:forsterite laser and the fundamental spectrum of the Ti:sapphire laser has given us access to $F_{0}$, the carrier-envelope offset frequency of the combined frequency comb. Using techniques of nonlinear frequency conversion, our approach has great potential for phase-coherent optical frequency synthesis from the ultraviolet through the midinfrared.

We thank the Bureau International des Poids et Mesures for the loan of a pump laser and Kristan Corwin for helpful discussions. A. Bartels's e-mail address is albrecht@boulder.nist.gov.

\section{References}

1. D. J. Jones, S. A. Diddams, J. K. Ranka, A. Stentz, R. J. Windeler, J. L. Hall, and S. T. Cundiff, Science 288, $635(2000)$.

2. U. Morgner, R. Ell, G. Metzler, T. R. Schibli, F. X. Kärtner, J. G. Fujimoto, H. A. Haus, and E. P. Ippen, Phys. Rev. Lett. 86, 5462 (2001).

3. T. M. Ramond, A. Bartels, S. A. Diddams, and L. Hollberg, Opt. Lett. 27, 1842 (2002).

4. A. Baltuska, Th. Udem, M. Uiberacker, M. Hentschel, E. Goulielmakis, Ch. Gohle, R. Holzwarth, V. S. Yakovlev, A. Scrinzi, T. W. Hänsch, and F. Krausz, Nature 421, 611 (2003).

5. Th. Udem, R. Holzwarth, and T. W. Hänsch, Nature 416, 233 (2002).

6. R. K. Shelton, L.-S. Ma, H. C. Kapteyn, M. M. Murnane, J. L. Hall, and J. Ye, Science 293, 1286 (2001).

7. D. J. Jones, K. W. Holman, M. Notcutt, Y. Ye, J. Chandalia, L. A. Jiang, E. P. Ippen, and H. Yokohama, Opt. Lett. 28, 813 (2003).

8. J. B. Schlager, B. E. Callicoatt, R. P. Mirin, N. A. Sanford, D. J. Jones, and J. Ye, Opt. Lett. 28, 2411 (2003).

9. W. Seitz, T. R. Schibli, U. Morgner, F. X. Kärtner, C. H. Lange, W. Richter, and B. Braun, Opt. Lett. 27, 454 (2002).

10. Z. Wei, Y. Kobayashi, Z. Zhang, and K. Torizuka, Opt. Lett. 26, 1806 (2001).

11. T. R. Schibli, J. Kim, O. Kuzucu, J. T. Gopinath, S. N. Tandon, G. S. Petrich, L. A. Kolodziejski, J. G. Fujimoto, E. P. Ippen, and F. X. Kärtner, Opt. Lett. 28, 947 (2003).

12. J. Rauschenberger, T. M. Fortier, D. J. Jones, J. Ye, and S. T. Cundiff, Opt. Express 10, 1404 (2002), http:// www.opticsexpress.org.

13. K. W. Holman, D. J. Jones, J. Ye, and E. P. Ippen, Opt. Lett. 28, 2405 (2003).

14. Y. Kobayashi, K. Torizuka, and Z. Wei, Opt. Lett. 28, 746 (2003).

15. A. Bartels and H. Kurz, Opt. Lett. 27, 1839 (2002).

16. I. Thomann, A. Bartels, K. L. Corwin, N. R. Newbury, L. Hollberg, S. A. Diddams, J. W. Nicholson, and M. F. Yan, Opt. Lett. 28, 1368 (2003).

17. A. Bartels, S. A. Diddams, T. M. Ramond, and L. Hollberg, Opt. Lett. 28, 663 (2003). 